\title{
1 Trace Analysis of Surfactants in Corexit Oil Dispersant Formulations and Seawater
}

2 Benjamin J. Place ${ }^{1, \dagger}$, Matt J. Perkins ${ }^{2}$, Ewan Sinclair ${ }^{3}$, Adam L. Barsamian ${ }^{1}$, Paul R.

3 Blakemore $^{1}$, Jennifer A. Field ${ }^{2, *}$

$4 \quad{ }^{1}$ Department of Chemistry, Oregon State University, Corvallis, OR

$5 \quad{ }^{2}$ Department of Environmental and Molecular Toxicology, Oregon State University, Corvallis,

$6 \quad$ OR

$7 \quad{ }^{3}$ College of Osteopathic Medicine, Touro University-California, Vallejo, CA

8

$9 *$ * Corresponding Author Information:

10 Mailing Address: Department of Environmental and Molecular Toxicology, 1007 ALS Building,

11 Oregon State University, Corvallis, OR 97331

12 Email: Jennifer.Field@ oregonstate.edu

13 Phone: 541-737-2265

14 Fax: 541-737-0497

15

$16{ }^{\dagger}$ Current Address: National Institute of Standards and Technology, 100 Bureau Dr., Mail Stop

17 8392, Gaithersburg, MD 20899 


\section{Abstract}

21 After the April 2010 explosion on the Deepwater Horizon oil rig, and subsequent release of

22 millions of barrels of oil, two Corexit oil dispersant formulations were used in unprecedented

23 quantities both on the surface and sub-surface of the Gulf of Mexico. Although the dispersant

24 formulations contain four classes of surfactants, current studies to date focus on the anionic

25 surfactant, bis-(2-ethylhexyl) sulfosuccinate (DOSS). Factors affecting the integrity of

26 environmental and laboratory samples for Corexit analysis have not been systematically

27 investigated. For this reason, a quantitative analytical method was developed for the detection of

28 all four classes of surfactants, as well as the hydrolysis products of DOSS, the enantiomeric

29 mixture of $\alpha$ - and $\beta$-ethylhexyl sulfosuccinate ( $\alpha$-/ $\beta$-EHSS). The analytical method was then

30 used to evaluate which practices for sample collection, storage, and analysis resulted in high

31 quality data. Large volume, direct injection of seawater followed by liquid chromatography

32 tandem mass spectrometry (LC-MS/MS) minimized analytical artifacts, analysis time, and both

33 chemical and solid waste. Concentrations of DOSS in the seawater samples ranged from $71-$

$3413,000 \mathrm{ng} / \mathrm{L}$, while the nonionic surfactants including Span 80, Tween 80, Tween 85 were

35 detected infrequently (26\% of samples) at concentrations from $840-9100 \mathrm{ng} / \mathrm{L}$. The

36 enantiomers $\alpha-/ \beta$-EHSS were detected in seawater, at concentrations from $200-1,900 \mathrm{ng} / \mathrm{L}$, and

37 in both Corexit dispersant formulations, indicating $\alpha$-/ $\beta$-EHSS were applied to the oil spill and

38 may be not unambiguous indicator of DOSS degradation. Best practices are provided to ensure

39 sample integrity and data quality for environmental monitoring studies and laboratory that

40 require the detection and quantification of Corexit-based surfactants in seawater. 


\section{Introduction}

In response to the Deepwater Horizon oil rig explosion, and subsequent release of oil into

43 the Gulf of Mexico, an unprecedented quantity of oil dispersant was applied to both the surface

44 oil slick and at the wellhead in order to mitigate the environmental impact of the oil spill

45 (Operational Science Advisory Team, 2010). During the spill 7 million liters of Corexit 9500 and 9527 oil dispersant was applied, 4.1 million liters was applied to the surface while 2.9 million

47 was applied sub-surface (National Commission on the BP Deepwater Horizon Oil Spill and

48 Offshore Drilling, 2011). Multiple studies show low to moderate toxicity of Corexit oil

49 dispersants, both as the dispersant alone and when mixed with crude oil (US EPA, 2011;

50 Anderson et al., 2009; George-Ares and Clark, 2000; Goodrich et al., 1991; Wooten et al., 2012).

51 The environmental impact of the application of oil dispersant at these unprecedented volumes is

52 unknown.

Analytical tools are necessary to study the environmental distribution and fate of the oil

54 dispersant constituents in the Gulf of Mexico. In addition, these tools are necessary to support

55 laboratory studies on dispersant components such as toxicity testing and biodegradation

56 experiments. Prior to the spill, there were few analytical methods available for these purposes

57 (Place et al., 2010), primarily due to the fact that the constituents of both Corexit dispersants

58 were proprietary. After the spill, the United State Environmental Protection Agency (US EPA)

59 reported the components of the oil dispersants, which included four surfactants: bis-(2-

60 ethylhexyl) sulfosuccinate (DOSS), sorbitan monooleate (Span 80), sorbitan monooleate

61 polyethoxylate (Tween 80), and sorbitan trioleate polyethoxylate (Tween 85) (US Environmental

62 Protection Agency, 2011). In addition, the US EPA set the aquatic life benchmark for chronic

63 exposure of DOSS to 40,000 ng/L and a reporting limit of 20,000 ng/L (benchmarks were not set 
64 for the other surfactant components of Corexit dispersants) (Operational Science Advisory Team,

65 2010). Since 2010, multiple analytical methods that have been developed in order to detect levels

66 of Corexit oil dispersants in Gulf of Mexico seawater, although these studies mainly focused on

67 DOSS as the indicator for the presence of Corexit 9500 and 9527.(Hayworth and Clement, 2012;

68 Kujawinski et al., 2011; Mathew et al., 2012; Ramirez et al., 2013) To the best of our knowledge,

69 analytical methods for nonionic surfactants, including Tween 80 and Tween 85,(Crescenzi et al.,

70 1995; Petrovic and Barceló, 2001; Petrovic et al., 2002) have not been developed for seawater

71 analysis. To fully characterize the complex mixture of the dispersant formulations, analytical

72 methods are needed for detecting all the dispersant constituents which exhibit varying chemical

73 properties.

There is little information about the fate of these dispersants in aquatic environments. The

75 chemical and biological transformation pathways, and the resultant toxicity of these

76 transformation products, have not been characterized. However, Hales (1993) proposed the

77 biodegradation pathway of linear dialkyl sulfosuccinates and others have reported the presence

78 of the hydrolysis products of DOSS, $\alpha$-/ $\beta$-ethylhexyl sulfosuccinate ( $\alpha$-/ $\beta$-EHSS) (Campo et al.,

79 2013). In addition to being degradation products, $\alpha$-/ $\beta$-EHSS can occur potentially as

80 intermediates in the synthesis of DOSS. Analytical methods need to be developed to detect and

81 track these degradation products, as well as the parent compounds in the dispersant, in order to

82 better understand the environmental fate of the dispersants. However, at present, no

83 commercially-available standards exist for $\alpha$-/ $\beta$-EHSS nor are there isotopically-labeled internal

84 standards for $\alpha$ - $/ \beta$-EHSS.

85 The objective of this study was to develop an analytical method for the quantitative

86 detection of the surfactants components in seawater, as well as investigate the complexities of 
87 sample collection, handling, and storage. Current methods to date use sample preparation steps

88 such as solid-phase extraction (Kujawinski et al., 2011; Ramirez et al., 2013) or direct injection

89 after sample dilution (Mathew et al., 2012; Ramirez et al., 2013). Large-volume injection liquid

90 chromatography (LVI-LC) is an alternative to solid-phase extraction that has been demonstrated

91 for environmental contaminants in surface water and wastewater systems (Backe et al., 2011;

92 Busetti et al., 2012; Chiaia et al., 2008), but not yet for seawater. The instrumental method

93 utilizes large-volume injection liquid chromatography (LVI-LC) with mass spectrometry for a

94 sensitive analytical method capable of analyzing seawater for all surfactant components in

95 Corexit dispersants with minimal sample preparation. In addition to the chemical components in

96 the oil dispersant mixtures, an analytical standard for $\alpha$-EHSS and its ${ }^{13} \mathrm{C}$-labeled analog were

97 synthesized for use in quantifying $\alpha-/ \beta$-EHSS. Best practices to ensure sample integrity and data

98 quality during sample collection, handling, and storage were developed and validated. The

99 capabilities of this analytical method were then demonstrated by the analysis of select seawater

100 samples and Corexit commercial formulations.

101

102

\section{Methods and materials}

\section{$103 \quad 2.1$ Chemicals and Standards}

$104 \quad$ 2.1.1 Analytical Standards

A pure (98.1\%) solid standard of bis-(2-ethylhexyl) sodium sulfosuccinate (DOSS) was

106 obtained from Sigma Aldrich (Saint Louis, MO). Liquid standards of sorbitan monooleate (Span

107 80; purity: 70.5\%), sorbitan monooleate polyethoxylate (Tween 80; purity: 74\%), and sorbitan

108 monooleate polyethoxylate (Tween 85; purity: 67\%) were obtained from Sigma Aldrich (St. 
109 Louis, MO). A standard containing ${ }^{13} \mathrm{C}_{4}$-labeled DOSS was provided by Ed Furlong and James

110 Gray at the United States Geological Survey National Water Quality Laboratory (Denver, CO)

111 that was synthesized by Cambridge Isotope Laboratories, Inc (Andover, MA). Quantitative

112 standards for the DOSS hydrolysis products, $\alpha$ - and $\beta$-ethylhexyl sulfosuccinate ( $\alpha$-/ $\beta$-EHSS)

113 were synthesized in laboratory as described below.

114 HPLC-grade isopropanol, acetonitrile, acetone, and methanol were purchased from

115 Sigma Aldrich. Laboratory 18-M $\Omega$, deionized (DI) water was obtained by an in-house Millipore

116 Synergy unit with an LC-Pak polisher (EMD Millipore Corp, Billerica, MA). High purity

117 ammonium acetate was also purchased from Sigma Aldrich. Instant Ocean® salt mix (Spectrum

118 Brands Company, Madison, WI) was provided by Robert Tanguay at Oregon State University.

119 Parent stock standards were prepared from solid or concentrate in solvent; DOSS

120 standards were prepared in methanol while Span 80, Tween 80, and Tween 85 were prepared in

121 isopropanol and $\alpha$-EHSS was prepared in deionized water. Although others report DOSS

122 standards are unstable in solution for longer than $24 \mathrm{~h}$ (Kujawinski et al., 2011), preliminary

123 work indicated that all solvent-based standards were stable for over 1.5 months at $4{ }^{\circ} \mathrm{C}$ (Figure

124 S5 in Supporting Information (SI)). Analytical standards were prepared in 25\% isopropanol

125 and $75 \%$ ocean salt solution (created by mixing $15.2 \mathrm{~g}$ of Instant Ocean $®$ in DI water). These

126 analytical standards were analyzed within 8 hours.

127

\subsection{2 $\alpha$-/ $\beta$-EHSS synthesis and purification}

$a$-EHSS [i.e., sodium 1-carboxy-2-(2-ethylhexyloxycarbonyl)ethanesulfonate] was

130 prepared from maleic anhydride by the method of Baczko et al. (2001) and this same approach

131 was applied to $\left[{ }^{13} \mathrm{C}\right]_{4}$-maleic anhydride to create $\left[{ }^{13} \mathrm{C}\right]_{4}-a$-EHSS. Both compounds were isolated 
132 as colorless powders by precipitation of their disodium salts and the unlabeled material was

133 quantified by ${ }^{1} \mathrm{H}$ NMR spectral analysis (700 MHz, $\left.\mathrm{D}_{2} \mathrm{O}-\mathrm{CD}_{3} \mathrm{OD}\right)$ using 4-

134 (dimethylamino)benzaldehyde as an internal standard (powder was $2.152 \mu \mathrm{mol} / \mathrm{mg}$ in $a$-EHSS

135 with remainder inorganic sodium salts). $\beta$-EHSS was prepared via a three-step sequence from

136 maleic anhydride that comprised of alcoholysis with 4-methoxybenzyl alcohol, $N, N^{\prime}-$

137 dicyclohexyldiimide (DCC) coupling of the resulting monoester with ( \pm )-2-ethylhexan-1-ol, and

138 selective removal of the 4-methoxybenzyl group from the mixed diester by treatment with

139 trifluoroacetic acid. Details for this synthetic chemistry will be reported elsewhere.

\section{$141 \quad$ 2.2 Best Practices: Sample Handling and Storage}

\section{$142 \quad$ 2.2.1 Analytical Standard Stability}

143 Standards made from pure solid (for DOSS and $\alpha$-/ $\beta$-EHSS) or liquid (for Span 80,

144 Tween 80, and Tween 85) were made in 25-mL volumetric flasks with methanol (for DOSS), DI

145 water (for $\alpha$-/ $\beta$-EHSS), or isopropanol (for Span 80, Tween 80, and Tween 85). Standards were

146 made at three different dates to compare the long term stability of the stock and stored at $4{ }^{\circ} \mathrm{C}$

147 until analysis. Standards from multiple long-term time points were compared to standards made

148 on the day of analysis. Working standards, consisting of $25 \%$ isopropanol and $75 \%$ Instant

149 Ocean or $100 \%$ Instant Ocean, were made in multiple 6-mL glass autosampler vials and analyzed 150 over time while left at $4{ }^{\circ} \mathrm{C}$ (room temperature $\alpha$-/ $\beta$-EHSS) for on the autosampler tray. Each 151 solvent system was analyzed at least 4 times over 12 hours. 
To determine short-term storage stability (13 h), samples containing all analytes were

155 made in $100 \%$ Oregon Coast seawater at the second lowest concentration level in order to

156 simulate samples taken for environmental monitoring. The samples were stored in 50-mL HDPE

157 centrifuge tubes and three samples of each standard were stored at room temperature $\left(20{ }^{\circ} \mathrm{C}\right), 4$

$158{ }^{\circ} \mathrm{C}$, and at $-20{ }^{\circ} \mathrm{C}$. For each treatment, one sample at each of three time points over $13 \mathrm{~h}$ was

159 prepared as described below in Section 2.3 and analyzed in triplicate.

160 To determine the long-term stability of seawater samples, open ocean water collected

161 from the Oregon coast was spiked with all analytes and the mixture was separated into multiple

$16250-\mathrm{mL}$ centrifuge tubes. All long-term stability samples were then stored at $-20{ }^{\circ} \mathrm{C}$ until analysis.

163 During each analysis, for a total of 7 months, individual samples were thawed in the presence of

164 isopropanol, as described below in Section 2.4.

165

166

\subsection{Corexit Formulation Analysis}

In order to determine the concentration of each analyte in the whole Corexit 9500 and

1689527 commercial formulations (donated by Ronald Tjeerdema of the University of California at

169 Davis) were diluted in methanol. Then, analytical samples were made in 25\% isopropanol:75\%

170 Instant Ocean at nominally $1 \mathrm{mg} / \mathrm{L}$ and $100 \mu \mathrm{g} / \mathrm{L}$ total Corexit concentrations. The samples were

171 prepared in the Instant Ocean solution in order to include the formulation analysis within the

172 seawater analysis (as standards were made in Instant Ocean to mimic ion suppression). The

173 higher concentration was used to determine $\alpha$-/ $\beta$-EHSS concentrations and the lower

174 concentration was used to determine surfactant concentrations. All samples were analyzed using

175 the same method as for field samples as described below. 


\subsection{Field Sample Collection and Preparation}

Gulf of Mexico seawater samples were collected on the R/V Walton Smith between May

17925,2010 and June 6, 2010. The samples were collected by a CTD-Niskin rosette system at

180 multiple sites and varying depths.(Joye et al., 2011) The collected water was then split into BD

181 Falcon 50-mL polypropylene centrifuge tubes (BD Biosciences, San Jose, CA) and frozen

182 immediately. The samples were kept frozen until they were shipped with blue ice to Oregon

183 State University. Field blanks consisting of laboratory water (using a MilliQ Advantage A10

184 water purification system) were made on the ship and frozen until shipment. Samples were

185 shipped frozen and stored at -20 C upon receipt.

186 To reduce or eliminate analyte loss, the frozen seawater samples (in the $50 \mathrm{~mL}$ centrifuge

187 vials) were first weighed to determine volume, and then transferred (while frozen) into a $250 \mathrm{~mL}$

188 HDPE bottle. The centrifuge vials were then rinsed with 3 aliquots of isopropanol (final

189 isopropanol volume equivalent to $25 \%$ of the final sample volume) and the rinsate was added to

190 the frozen seawater sample in the $250 \mathrm{~mL}$ bottle. Field sample preparation steps significantly

191 impacted the recovery of analytes from the seawater samples. Prior to analysis, $5 \mathrm{~mL}$ aliquots of

192 seawater sample/isopropanol were transferred to a $6 \mathrm{~mL}$ glass autosampler vial and spiked with

193 labeled internal standard solutions. For this study, no autosampler vial caps were used because

194 they were identified as a potential source of DOSS contamination.

$196 \quad 2.5$ Instrumental Analysis

$197 \quad$ 2.5.1 Large-Volume Injection Liquid Chromatography with Tandem Mass Spectrometry

198 (LVI-LC-MS/MS) 
200 (Agilent Technologies, Inc., Santa Clara, CA). The HPLC was upgraded with large volume

201 injection and multidraw kits for injecting volumes up to 1,800 $\mu \mathrm{L}$. An Agilent Zorbax C18 guard 202 column (4.6 mm ID x $12.5 \mathrm{~mm}$ length x 5- $\mu \mathrm{m}$ particle size) was placed in front of a Targa C18

203 analytical column (2.1 mm ID x $150 \mathrm{~mm}$ x 5- $\mu$ m particle size; Higgins Analytical, Inc.,

204 Mountain View, CA). The guard column was replaced approximately every 100 injections.

205 Because the HPLC gave significant background levels of DOSS, an additional Agilent Zorbax

206 C18 guard column, with the same dimensions as described above, was placed in the flow path

207 after the solvent mixer and purge valve but prior to the autosampler as described by Powley et

208 al.(Powley et al., 2005) With this setup, DOSS contamination originating from within the HPLC 209 eluted after the DOSS analyte peak (Figure S7 in SI).

210 The HPLC mobile phase consisted $0.5 \mathrm{mM}$ ammonium acetate in DI water (A) and

211 acetonitrile (B). The gradient program followed a starting composition of 5\% B that was held for 212 the first $7 \mathrm{~min}$, increased to $50 \% \mathrm{~B}$ in $0.5 \mathrm{~min}$, increased to $60 \% \mathrm{~B}$ in $9.5 \mathrm{~min}$, followed by an

213 increase to $97.5 \% \mathrm{~B}$ that was then held for $10 \mathrm{~min}$ before the composition returned to $5 \% \mathrm{~B}$ in 1 $214 \mathrm{~min}$ for a total run time of $36 \mathrm{~min}$. In addition to the solvent gradient, the flow rate was 0.5

$215 \mathrm{~mL} / \mathrm{min}$ for the first $17 \mathrm{~min}$ before it was increased to $0.75 \mathrm{~mL} / \mathrm{min}$ for the rest of the analytical 216 run. In order to reduce solvent dwell time (the time it takes for changes in the gradient to reach 217 the analytical column) the autosampler switch valve was set to bypass the autosampler injector 218 system at $7 \mathrm{~min}$. To reduce analyte carryover, the autosampler switch valve switched back to 219 send the mobile phase through the injector system at $17.5 \mathrm{~min}$ (Figure S8 and S9 in SI).

220 Without this "main-pass" switch, nonionic analyte carryover ranged from $4-40 \%$ of the original 221 concentration. With the switch, the nonionic analytes retained in the injection system were 
222 pushed onto the column with the $97.5 \%$ acetonitrile mobile phase so that they eluted with the 223 analytes retained on the column.

224 To prevent fouling of the sample cones by the nonvolatile salts in seawater, the initial 225 flow from the column was diverted to waste, after $9.5 \mathrm{~min}$ the flow was switched to the mass 226 spectrometer. In addition, from 16 to $23.5 \mathrm{~min}$ the flow was diverted to waste during the injector 227 system cleaning step (the first 7.5 min of the main-pass switch). The entire LC-MS/MS timeline 228 is visually shown in SI.

229 Mass spectrometric detection for DOSS, Span 80, Tween 80, and Tween 85 was 230 performed with a Waters Acquity Triple Quadrupole Mass Spectrometer (Waters Corporation,

231 Milford, MA), while $\alpha$-/ $\beta$-EHSS was determined on a Waters Micromass Quattro Mass

232 Spectrometer. Two separate MS/MS systems were used rather than one because the $\alpha$-EHSS and 233 its ${ }^{13} \mathrm{C}_{4}-\alpha$-EHSS internal standard were synthesized after the surfactant analyses were complete. 234 All experiments were repeated to determine the analytical figures of merit for $\alpha$-EHSS but the 235 analyses were performed on an identical LVI-LC system that was interface with the Quattro 236 Micro MS/MS.

237 DOSS, ${ }^{13} \mathrm{C}_{4}$-DOSS, $\alpha$ - $/ \beta$-EHSS, and ${ }^{13} \mathrm{C}_{4}-\alpha$-EHSS were detected in negative ionization 238 mode with multiple reaction monitoring (MRM) mode. Span 80 was detected in positive 239 ionization mode with MRM mode with two MRM transitions. Tween 80 and Tween 85 represent 240 a homologous series of compounds with varying polyethoxylate chain lengths and therefore 241 could not be identified by a single MRM transition. Alternatively, a common fragment ion $(\mathrm{m} / \mathrm{z}$ 242 309) was identified for both Tween 80 and Tween 85, as reported by Borisov et al.(Borisov et 243 al., 2011) Therefore, precursor ion scanning (positive ionization) was used to scan for all 244 precursor masses $(\mathrm{m} / \mathrm{z}, 400-1300)$ that fragmented into $\mathrm{m} / \mathrm{z} 309$ in order to quantify the 
245 homologous series of Tween 80 and Tween 85. MS parameters and timeline for all analytes are 246 reported in the SI.

\subsubsection{Quantification and Quality Control}

Preliminary observations indicated that, even with a 95\% aqueous wash step, residual salts suppressed ionization of the nonionic analytes (Figure S5 in SI). Because internal standards

251 exist for DOSS and $\alpha$-/ $\beta$-EHSS, the ion suppression could be compensated for, but the nonionic 252 surfactants (Span 80, Tween 80, and Tween 85) do not have commercially available isotopically253 labeled internal standards and therefore the ion suppression can greatly impact quantification.

254 For purposes of compensating for the strong ion suppression of seawater, due to the high ionic 255 strength, all analytical standards were made in $25 \%$ isopropanol and $75 \%$ Instant Ocean for 256 matrix-matched calibration.

257 Calibration curves consisted of at least 5 calibration standards and required a correlation 258 coefficient of 0.99 or greater in order to be used for quantification. All calibration curves were $2591 / \mathrm{x}$ weighted, and standards whose calculated concentrations were beyond $30 \%$ of the intended 260 concentration were removed from the calibration curve calculation. Calibration curves spanned 261 from the lower limit of quantification (LLOQ) to the upper limit of quantification (ULOQ) for

262 DOSS (67-34,000 ng/L), $\alpha$-/ $\beta$-EHSS (150-25,000 ng/L), Span 80 (3,000-60,000 ng/L), Tween 80

263 (2,700-400,000 ng/L), and Tween 85 (700 -150,000 ng/L) (Table 1). Each calibration standard 264 was spiked to give a final concentration of $100 \mathrm{ng} / \mathrm{L}^{13} \mathrm{C}_{4}$-DOSS and $500 \mathrm{ng} / \mathrm{L}{ }^{13} \mathrm{C}_{4}-\alpha$-EHSS. 265 Blank and check standards were used for quality control purposes and consisted of at 266 least $20 \%$ of the total samples run in any given sequence. Check standards consisted of $25 \%$ 267 isopropanol:75\% Instant Ocean solution that was spiked with all analytes. For DOSS and $\alpha-/ \beta$ - 
268 EHSS quantification, the calculated concentration for the check standards were required to be

269 within $30 \%$ of the spiked concentration. For Span 80, Tween 80, and Tween 85 there were no

270 internal standards available; therefore, the check standard criteria required concentrations to be

271 within $35 \%$. Due to concerns about DOSS contamination, blanks, consisting of

272 isopropanol:Instant Ocean solution and spiked with ${ }^{13} \mathrm{C}_{4}$-DOSS, were used regularly to verify

273 that background DOSS concentration levels were below the LLOQ and that there was no

274 carryover of any of the analytes. Failure to meet QC criteria required corrective action until QC

275 checks were brought back into control before proceeding with sample analysis

276

$277 \quad$ 2.5.3 Method Performance Evaluation

278 To determine accuracy of the whole method, four samples of blank Oregon Coast

279 seawater were spiked with all analytes at low concentration levels (equivalent to the second

280 lowest standard). For $\alpha$-/ $\beta$-EHSS measurements, Oregon Coast seawater was spiked in the

281 absence of all other analytes at a concentration equivalent to the third lowest standard. Recovery

282 was determined as the ratio of calculated analyte concentration to spiked analyte concentration.

283 Precision was reported as the relative standard deviations (RSD) of the four replicate analyses

284 (Table 1).

285 In order to calculate limits of detection (LOD) and quantification, ten blank samples,

286 consisting of $25 \%$ isopropanol and $75 \%$ Oregon Coast seawater, were analyzed to determine a

287 baseline background signal (i.e. noise) for all of the analytes. The area of the background signal

288 for each analyte was integrated and a standard deviation of the area was calculated. A low-range

289 calibration curve spanning $\leq 2$ orders of magnitude for all analytes was then developed with

290 analytical standards prepared in $25 \%$ isopropanol and $75 \%$ Instant Ocean solution. The LOD and 
291 lower limit of quantification (LLOQ) were estimated by multiplying the background peak area

292 standard deviation by 3.3 and 10, respectively, and dividing this value by the slope of the low-

293 range calibration curve.(Health Canada, 1999)

\section{Results and Discussion}

\section{3.1 Analytical Method Performance}

297 To the best of our knowledge, this is the first study to quantitatively detect all surfactant

298 analytes of Corexit dispersant formulations in seawater. All analytes were chromatographically

299 separated (Figure 1) without adverse effects related to the direct injection of seawater. DOSS, $\alpha-$

$300 / \beta$-EHSS, and Span 80 are single compounds that could be identified using the common multiple

301 reaction monitoring (MRM) mode. The detection of the homologous series of Tween 80 and

302 Tween 85 was more challenging because the complex mixture of polyethoxylates made MRM

303 detection for each individual compound impractical. Furthermore, analytical standards for the

304 Tweens and Span are not commercially available. The precursor ion scanning technique, which

305 detected all mixture components that produce the $m / z, 309$ fragment ion, provided an alternative

306 to MRM for the detection of Tween 80 and Tween 85 (Figure S10 in SI).

307 LVI-LC is a tool for the sensitive detection of analytes in environmental aqueous samples

308 that avoids extensive sample preparation. The injection of non-volatile salts is of a concern for

309 any analytical method utilizing mass spectrometric detection as salt sprayed into the ionization

310 chamber can lead to sample cone fouling and corrosion. Utilizing the post-column divert valve

311 built into the mass spectrometer, the initial flow, containing most of the salt, was diverted to

312 waste away from the mass spectrometer. This was a vital step in the protection of the MS system 
313 during sample analysis. After months of analyses there was no significant deposition of salt on

314 the sample cones.

315 While column fouling is also a concern with large volume injection, a single analytical

316 column was used for approximately 1 year ( 2500 large volume injections) without observing

317 diminishing chromatographic peak quality. Guard columns could be used for approximately 100

318 injections before peak shape deterioration (primarily peak tailing and splitting). Even with the

319 above described instrumental protection procedures, ionization suppression was observed for the

320 nonionic analytes (Figure S5 in SI). We propose that the decrease in sensitivity is due to the

321 formation of sodium-adducted compounds, which result from low levels of residual salts that

322 retained with the analytes and co-eluted into the mass spectrometer. Sodium-adducted

323 compounds have been previously reported to decrease fragmentation efficiency.(Grimalt et al.,

324 2005; Pozo et al., 2008)

325

\subsubsection{Method Accuracy and Precision.}

Whole method accuracy, as indicated by percent recovery, ranged from $88-119 \%$

328 (Table 1). Whole method precision, as indicated by RSD, ranged from $1.4-23 \%$ (Table 1).

329 Higher RSD values were observed with Tween 85 (17\%) and Span 80 (23\%), which is due to the

330 poorer sensitivity to these compounds as well as the lack of an internal standard to accommodate

331 for between-injection differences in ionization efficiency. The developed method provides

332 similar recovery of DOSS $(88 \pm 10 \%$, mean $\pm 95 \% \mathrm{CI})$ as those for previously reported methods

333 (80 - 100\% recovery).(Gray et al., 2010; Kujawinski et al., 2011; Mathew et al., 2012) In

334 contrast, this LVI method required no sample preparation other than the addition of isopropanol, 
resulting in higher throughput of the present method. The addition of isopropanol, which ensured

336 analyte stability in seawater, was half the dilution than that employed by Mathew et al. (2012).

337 The use of ${ }^{13} \mathrm{C}_{4}$-DOSS as an internal standard for the nonionic compounds was evaluated

338 and the labeled compound did not adequately describe the variation of any of the nonionic

339 compounds, therefore it could not function as an internal standard for any Span 80 or the

340 Tweens. Future research examining the presence and fate of the nonionic analytes will require

341 analytical standards for the individual Tween 80 and Tween 85 polyethoxylate homologues and

342 isotopically-labeled internal standards for these analytes.

343 Recovery values for the nonionic analytes were better in the isopropanol:Instant Ocean

344 solution than in an ammonium acetate buffer solution, suggesting that the high salt content of the

345 seawater is the primary source of ion suppression and requires matrix-matched calibration (SI).

\section{3.1.3 Limits of Detection/Quantification}

348 Limits of detection (LOD) and lower limits of quantification (LLOQ) ranged from 16 to

349 1,300 ng/L and 67 to 3,000 ng/L, respectively (Table 1). The background contamination level of

350 DOSS had a mean estimated concentration of $10 \mathrm{ng} / \mathrm{L}$. Due to the high variability (130\% RSD)

351 of the DOSS background contamination the LOD was conservatively raised to be equal to the

352 LLOQ at $67 \mathrm{ng} / \mathrm{L}$ (Table 1). The use of laboratory blanks, travel blanks, and sample blanks were 353 extremely important eliminating sources of DOSS contamination, which were found to occur on 354 container surfaces and in organic solvents. The LOD for DOSS is higher than that reported by 355 Kujawinski et al. (2011) at $3 \mathrm{ng} / \mathrm{L}$ and Ramirez et al. (2013) at $7 \mathrm{ng} / \mathrm{L}$ (by SPE), although is 356 below other methods with detection limits of $440 \mathrm{ng} / \mathrm{L}$ (Ramirez et al., 2013), $250 \mathrm{ng} / \mathrm{L}$ (Gray et 357 al., 2010) and 20,000 ng/L (Mathew et al., 2012). Because comparable methods do not exist for 
$358 \alpha-/ \beta$-EHSS or the nonionic surfactants in seawater, comparisons of the LOD and LLOQs

359 obtained was not possible.

360 The sensitivity of DOSS and EHSS were multiple orders of magnitude better than those

361 of the nonionic analytes (Span 80, Tween 80, Tween 85). This is most likely due to the poorer

362 ionization efficiency and broader peak shape of the nonionic analytes. In addition, the peaks

363 designated as Tween 80 and Tween 85 represent a broad series of polyethoxylate compounds,

364 which results in a broader overall peak.

365

$366 \quad$ 3.1.4 Best Practices

367 3.1.4.1 Sources of DOSS Contamination

368 Gray et al. (2010) reported the presence of DOSS as a potential contaminant during

369 sample processing. During this study, multiple potential sources of DOSS were identified. These

370 sources included: incomplete cleaning of glassware, PTFE-coated autosampler septa, laboratory

371 deionized water (from three different DI water systems), and general handling of glassware.

372 Various procedures were established in order to eliminate and/or compensate for the DOSS

373 contamination sources. All glassware was cleaned with the following procedures: detergent soak

374 in laboratory tap water, rinsed with warm laboratory tap water, rinsed with laboratory DI water,

375 baked for 12 hours at $400 \mathrm{C}$, rinsed with methanol, and rinsed with $25 \%$ isopropanol/75\%

376 cleaned (see below) Instant Ocean solution. All Instant Ocean solutions were made by mixing

377 the commercial Instant Ocean salt with laboratory DI water and then mixed with ENVI-Carb

378 SPE bulk packing (Sigma Aldrich, Saint Louis, MO), using approximately $0.1 \mathrm{~g}$ ENVI-Carb per

$379100 \mathrm{~mL}$ of Instant Ocean solution. The solution was stirred for at least $1 \mathrm{hr}$ before it was vacuum

380 filtered and collected in a cleaned Erlenmeyer flask and stored at room temperature. All samples 
381 were put in cleaned $6 \mathrm{~mL}$ glass autosampler vials without septa. Due to the use of the pump

382 contamination column (see Section 2.5.1), DI water used for the mobile phase did not need to be 383 cleaned.

384 Care should be used for handling all samples to minimize sources of cross contamination, 385 including using clean glassware, minimizing the number of sample transfers, and changing 386 gloves regularly throughout the sample preparation process. The above procedures were all 387 found to minimize the presence of DOSS contamination in this laboratory, although DOSS

388 signals in blank controls were identified regularly. The use of blank controls for DOSS analytical 389 methods is extremely important in order to provide high-quality, quantitative data and low limits 390 of detection.

\section{$392 \quad$ 3.1.4.2 Analytical Standard Stability}

Parent stock standards were stable within an acceptable range over 44 days of analysis

394 when stored at $4{ }^{\circ} \mathrm{C}$ (Figure S3 in SI). It was therefore assumed that all standards would be 395 stable for long term storage in $100 \%$ organic solvent (water for $\alpha$-/ $\beta$-EHSS) when stored at the 396 designated temperature. The addition of 25\% isopropanol to Instant Ocean was necessary for the 397 stability of all analytes in the working standards (Figure S4 in SI).

\subsubsection{Seawater Sample Stability}

$400 \quad$ Initial experiments indicated rapid loss of all nonionic surfactants (not DOSS or EHSS) from 401 spiked ocean water when sitting at room temperature (Figure 2). Rinsing the HDPE vials with 402 isopropanol recovered DOSS but not the nonionic Span and Tweens. The recovery of DOSS is 403 attributed to desorption of DOSS from the HDPE vial but the lack of nonionic surfactant 
404 recovery may be due to biodegradation because the seawater had not been sterilized. The

405 addition of isopropanol may not only solubilize DOSS but it may also inhibit microbial activity,

406 thus ensuring the integrity of seawater samples containing Corexit components. Therefore, the

407 addition of isoproposal to recover DOSS and quench microbial activity was used to evaluate

408 three seawater sample storage temperatures including room temperature $\left(20{ }^{\circ} \mathrm{C}, 4{ }^{\circ} \mathrm{C}\right.$, and -20

$\left.409{ }^{\circ} \mathrm{C}\right)$.

410 The method of sample thawing into $100 \%$ isopropanol for the final sample composition

411 produced the most consistent results with full recovery of all analytes. If the loss was due to

412 biodegradation, the isopropanol sterilizes the solution and therefore ceases any further

413 biodegradation activity upon thawing.

414 For Tween 80 and Tween 85 , there were no significant changes in concentration after 7

415 months at $-20^{\circ} \mathrm{C}$ in seawater (as determined by the slope, $\mathrm{p}>0.05$ ). For Span 80 , there was a

416 significant negative slope $(\mathrm{p}<0.05)$ that would result in a $64 \%$ decrease in concentration over

417 the 7 months of analysis. For DOSS, there was a significant negative slope $(p<0.05)$ that would

418 result in a $21 \%$ decrease in concentration over the 7 months of analysis. These findings suggest

419 that while samples are stable for the short term when frozen at -20 C, long-term storage of these

420 samples can be detrimental to the quality of the data.

421

$422 \quad 3.2$ Method Demonstration

$423 \quad 3.2 .1$ Corexit 9500 and 9527 Formulations

$424 \quad$ Whole Corexit 9500 and Corexit 9527 formulations were determined to contain $18 \%$ and $42517 \%(w / w)$ DOSS, respectively. Both Corexit 9500 and Corexit 9527 contained detectable 426 quantities of $\alpha-/ \beta$-EHSS at $0.28 \%(\mathrm{w} / \mathrm{w})$ and $0.17 \%(\mathrm{w} / \mathrm{w})$, respectively. It was beyond the scope 
427 of the current study to determine whether the presence of $\alpha$-/ $\beta$-EHSS was due to synthetic

428 impurities or the degradation of DOSS during storage of the Corexit formulations. The nonionic

429 surfactants were detected in the Corexit 9500 at $4.4 \%$ (w/w, Span 80), 18\% (w/w, Tween 80),

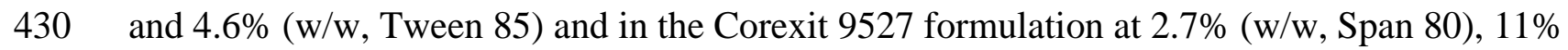

431 (w/w, Tween 80), and 4.3\% (w/w, Tween 85). It should be noted that these concentrations may

432 vary between batches and the reported values may not be representative of all Corexit

433 formulations used in the Gulf.

\subsubsection{DOSS in Gulf of Mexico Seawater}

436 Quantifiable concentrations of DOSS were detected in over half of the seawater samples

437 analyzed, with concentrations ranging from 71 to $13,000 \mathrm{ng} / \mathrm{L}$ (Table 2). The majority of the

438 samples containing detectable DOSS concentrations were at depths deeper than or equal to 1,000

$439 \mathrm{~m}$, with a mean concentration of $4,100 \mathrm{ng} / \mathrm{L}(\mathrm{n}=8)$. The mean concentration at the more shallow

440 depths was $110 \mathrm{ng} / \mathrm{L}(\mathrm{n}=4)$. The measured DOSS concentrations of depth seawater samples are

441 consistent with those previously reported by Kujawinski et al. (2011) and are at concentrations

442 below the detection limits reported by Mathew et al. (2012).

\section{$444 \quad 3.2 .3 \alpha-/ \beta-E H S S$ in Gulf of Mexico Seawater}

There were multiple detections of $\alpha-/ \beta$-EHSS that were above the LOD of $16 \mathrm{ng} / \mathrm{L}(\mathrm{n}=15$;

446 Table 2) in the analyzed seawater. Quantifiable concentrations of $\alpha$-/ $\beta$-EHSS were detected in 3

447 samples with a concentration range from $200-1900 \mathrm{ng} / \mathrm{L}$. Although most $\alpha-/ \beta$-EHSS detections

448 correspond with DOSS detections, there were samples that contained detectable quantities of $\alpha-/$

$449 \quad \beta$-EHSS without DOSS and vice versa. 
While the other analytes portrayed sample stability issues in laboratory seawater

451 standards, $\alpha-/ \beta$-EHSS compounds did not display any loss of concentration in seawater. This

452 observation suggests that $\alpha$-/ $\beta$-EHSS are more water soluble and will be in the aqueous phase

453 longer than any of the parent analytes. Because detectable quantities of $\alpha-/ \beta$-EHSS were

454 observed in the Corexit formulations, the detection of $\alpha-/ \beta$-EHSS in seawater cannot be used as

455 an unambiguous indicator of DOSS degradation in the environment. $\alpha$-EHSS, but not $\beta$-EHSS,

456 was also at detectable levels in DOSS analytical standards, most likely as an synthetic impurity

457 (approximately 400 ppm concentration in the solid DOSS standard). Therefore, care needs to be

458 taken when analyzing laboratory samples from toxicity or biodegradation studies for $\alpha$-/ $\beta$-EHSS

459 because it occurs in Corexit and in DOSS analytical standards.

\subsubsection{Nonionic Compounds in Gulf of Mexico Seawater}

462 There were no detectable quantities of Span 80 in any of the analyzed samples (Table 2).

463 Samples that were positive for the nonionic analytes contained concentrations for Tween 80 that

464 ranged from 3,500 to 9,100 ng/L (n=4) and Tween 85 that ranged from 840 to 2,900 ng/L (n=3,

465 Table 2). There was no significant correlation between concentrations of Tween 80 and Tween

46685 (correlation coefficient $r^{2}=0.48, n=6$ ). While there was a greater number of analyte detections

467 observed at the lower depths, the purpose of the sampling program was not to obtain sufficient

468 monitoring data to develop a correlation between depth and analyte concentration.

469 The difficulty of stabilization of the nonionic compounds in seawater, combined with their

470 relatively high LLOQs, is consistent with the relatively few observations of the nonionic analytes

471 in seawater. The degradation of the nonionic surfactants in various conditions has been

472 previously reported by many researchers and this is consistent with the rapid loss of the nonionic 
473 analytes in non-sterilized laboratory seawater (Kerwin, 2008). Others have found that the rapid

474 biological loss of sorbitan polyethoxylates, such as Tween 80 and Tween 85 , due to the

475 degradation by esterase enzymes.(Tellingen et al., 1999) This is consistent with the rapid loss of

476 the nonionic analytes in non-sterilized laboratory seawater.

\section{Conclusions}

The analytical protocol used in this study provides a sensitive and rugged method for the 480 detection and quantification of the multiple surfactant components in Corexit oil dispersant in 481 seawater samples. The analyte stability findings suggest that protocols for sample handling and 482 instrumental analysis can greatly impact the quality of the data produced. A more thorough, and 483 more current, set of Gulf of Mexico seawater samples (both surface and at depth) would provide 484 a better understanding of the spatial distribution of the surfactants. In addition, future studies to 485 determine the chemical and bio-degradation of DOSS for the formation of $\beta$-EHSS and $\alpha$-EHSS, 486 as well as degradation of the nonionic surfactants, are necessary to determine the environmental 487 implications of these measurements.

4895 Acknowledgements

490 The authors would like to thank James Gray and Ed Furlong (USGS) for collaborative 491 discussions and provision of the ${ }^{13} \mathrm{C}_{4}$-DOSS standard; Samantha Joye and Kim Hunter (UGA) 492 for the collection and provision of Gulf of Mexico seawater samples; and Ronald Tjeerdema 493 (UCD) for early collaborations related to Corexit oil dispersant and donation of the Corexit 9500 494 and 9527 formulation. Gulf of Mexico sample collection, aboard the R/V Walton Smith, was 495 supported by a grant from the National Science Foundation OCE-1043224 given to Samantha B. 
496 Joye. In addition, this study was supported, in part, by a grant from the BP/The Gulf of Mexico 497 Research Initiative (as part of the University of Mississippi-led consortium for research entitled 498 'Ecosystem Impacts of Oil and Gas Inputs to the Gulf (ECOGIG)') contribution number 217, the 499 OSU Superfund Research Program Award Number P42ES016465 from the National Institute of 500 Environmental Health Sciences, and the N.L. Tartar Research Fellowship from Oregon State

501 University. Research reported in this publication was supported by the National Institute of 502 Environmental Health Sciences of the National Institutes of Health under Award Number 503 T32ES007060. The content is solely the responsibility of the authors and does not necessarily 504 represent the official views of the National Institutes of Health. 
506 Table 1. Whole method performance indicated by limit of detection (LOD), lower limit of 507 quantification (LLOQ), upper limit of quantification (ULOQ), recovery, precision, and the 508 method for individual analyte quantification.

\section{Recovery}

\begin{tabular}{lcccccc} 
& LOD & LLOQ & ULOQ & $\mathbf{( \% \pm 9 5 \%}$ & RSD & Quantification \\
Compound & $(\mathbf{n g} / \mathbf{L})$ & $(\mathbf{n g} / \mathbf{L})$ & $(\mathbf{n g} / \mathbf{L})$ & $\mathbf{C I})$ & $\mathbf{( \% )}$ & Method \\
\hline DOSS & $67^{*}$ & 67 & 34,000 & $88 \pm 10$ & 10 & ISC: ${ }^{13} \mathrm{C}_{4}$-DOSS \\
$\alpha$-/ $\beta$-EHSS & $16 \dagger$ & $150 \dagger$ & 25000 & $98 \pm 6$ & 1.4 & ISC: ${ }^{13} \mathrm{C}_{4}-\alpha$-EHSS \\
Span 80 & 1,250 & 3,000 & 60,000 & $91 \pm 21$ & 23 & Ext. Cal \\
Tween 80 & 987 & 2,700 & 400,000 & $119 \pm 13$ & 10 & Ext. Cal \\
Tween 85 & 99 & 700 & 150,000 & $106 \pm 20$ & 17 & Ext. Cal
\end{tabular}

$510 *$ DOSS LOD is equal to DOSS LOQ due to background variability. $\dagger$ ISC: ${ }^{13} \mathrm{C}_{4}$-DOSS -

511 internal standard calibration using ${ }^{13} \mathrm{C}_{4}$-DOSS as internal standard; ISC: ${ }^{13} \mathrm{C}_{4}-\alpha$-EHSS - internal 512 standard calibration using ${ }^{13} \mathrm{C}_{4}-\alpha$-EHSS as an internal standard; and Ext. Cal. - external standard 513 calibration 
515 Figure 1. An LVI-HPLC/MSMS chromatogram of all analytes in an analytical standard

516 consisting of $25 \%$ isopropanol and $75 \%$ Instant Ocean.
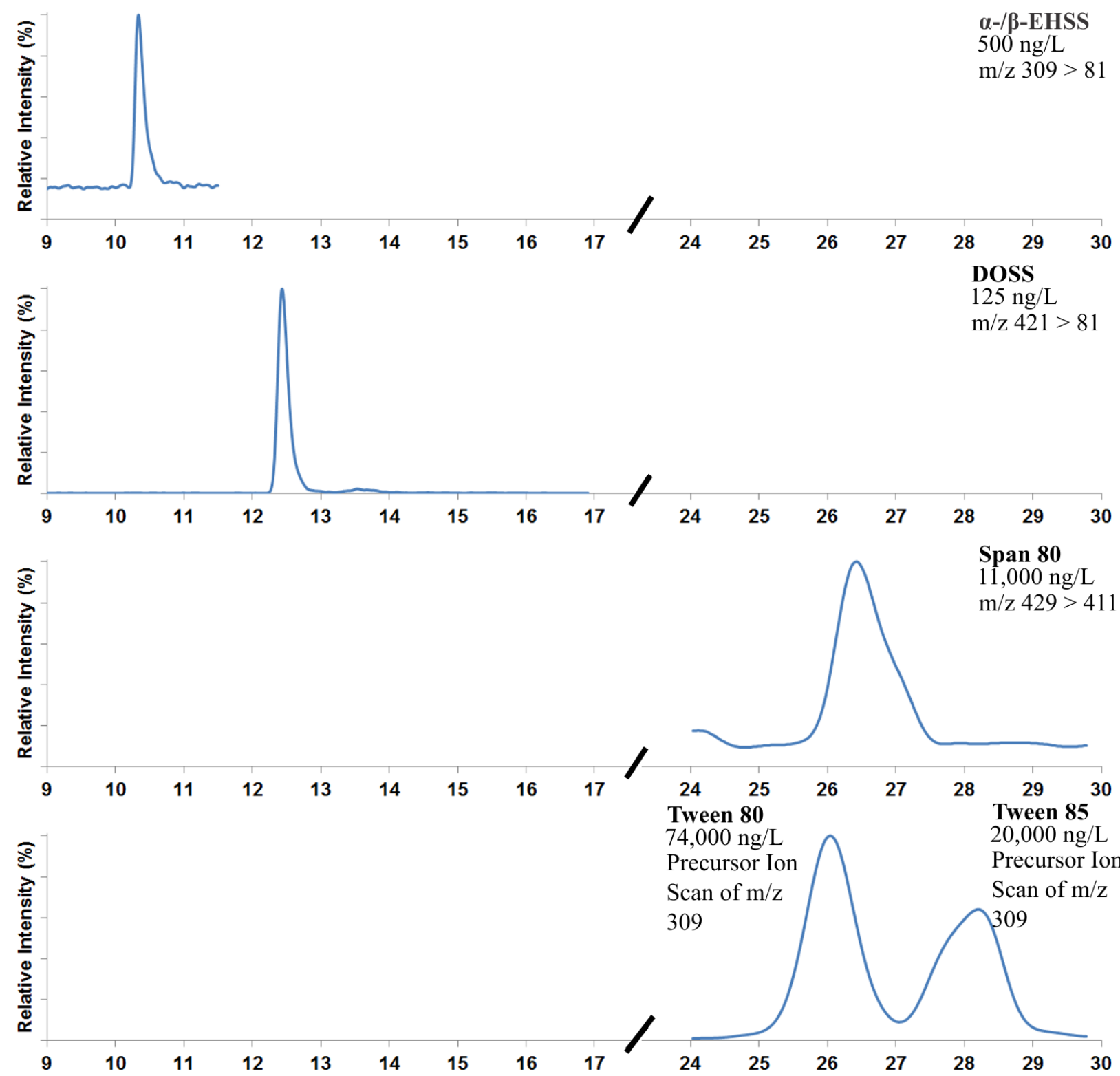

$125 \mathrm{ng} / \mathrm{L}$

$\mathrm{m} / \mathrm{z} 421>81$

Span 80 $\mathrm{m} / \mathrm{z} 429>411$ 
Figure 2. Short-term stability $(\leq 13 \mathrm{~h})$ of analytes in seawater in HDPE centrifuge tubes at various temperatures. After storage but prior to analysis, all samples were transferred to a 250 $\mathrm{mL}$ HDPE bottle and the centrifuge tube was rinsed with isopropanol and more isopropanol was added to give $25 \% \mathrm{v} / \mathrm{v}$. Analyte concentrations (C) were normalized to initial concentrations

$523\left(\mathrm{C}_{\mathrm{o}}\right)$.

524
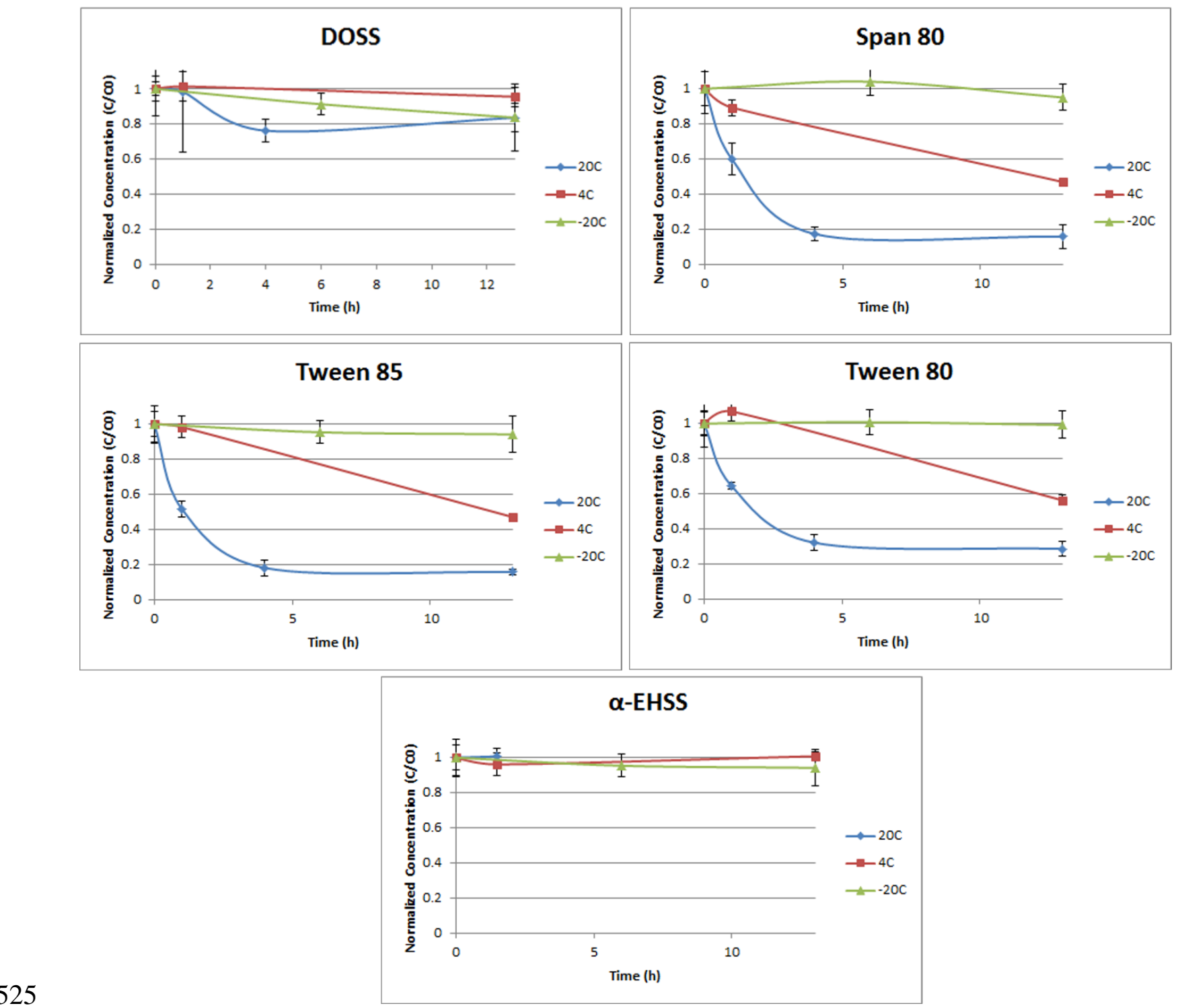
527 Table 2. Concentrations of DOSS, $\alpha$-/ $\beta$-EHSS, Span 80, Tween 80, and Tween 85 for each

528 sampling location, with sample conditions of depth and distance from the Deepwater Horizon

529 well head (designated MC252).

530

\section{Distance}

to MC252

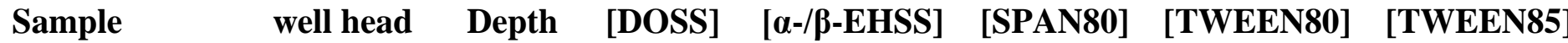

\begin{tabular}{|c|c|c|c|c|c|c|c|c|}
\hline Station & Cast & (m) & (m) & $n g / L$ & $\mathrm{ng} / \mathrm{L}$ & $n g / L$ & $n g / L$ & $n g / L$ \\
\hline \multirow[t]{4}{*}{ WS58 } & 75 & 410 & 600 & nd & nd & nd & nd & nd \\
\hline & & & 900 & nd & nd & nd & nd & nd \\
\hline & & & 1210 & 7,700 & $<$ LLOQ & nd & nd & 860 \\
\hline & & & 1400 & nd & nd & nd & nd & nd \\
\hline WS6 & 73 & 610 & 1180 & 13,000 & $<$ LLOQ & nd & 4,800 & 840 \\
\hline \multirow[t]{4}{*}{ WS76 } & 86 & 1290 & 50 & nd & $<$ LLOQ & nd & nd & nd \\
\hline & & & 1000 & nd & nd & nd & 9,100 & nd \\
\hline & & & 1100 & 100 & nd & nd & 5,900 & nd \\
\hline & & & 1200 & 11,000 & $<$ LLOQ & nd & nd & 2,900 \\
\hline \multirow[t]{3}{*}{ WS78 } & 90 & 13320 & 90 & nd & 1900 & nd & nd & nd \\
\hline & & & 600 & 95 & 530 & nd & nd & nd \\
\hline & & & 1130 & nd & $<$ LLOQ & nd & nd & nd \\
\hline \multirow[t]{5}{*}{ WS79 } & 91 & 15790 & 90 & 71 & $<$ LLOQ & nd & nd & nd \\
\hline & & & 90 & 110 & nd & nd & nd & nd \\
\hline & & & 600 & nd & $<$ LLOQ & nd & 3,500 & nd \\
\hline & & & 900 & 76 & $<$ LLOQ & nd & nd & nd \\
\hline & & & 1050 & 170 & NA & nd & nd & nd \\
\hline \multirow[t]{6}{*}{ WS16 } & 89 & 17700 & 100 & nd & $<$ LLOQ & nd & nd & nd \\
\hline & & & 600 & 170 & $<$ LLOQ & nd & nd & nd \\
\hline & & & 1025 & 76 & $<$ LLOQ & nd & nd & nd \\
\hline & & & 1100 & nd & 200 & nd & nd & nd \\
\hline & & & 1200 & 220 & $<$ LLOQ & nd & nd & nd \\
\hline & & & 1300 & 200 & nd & nd & nd & nd \\
\hline
\end{tabular}

531 LLOQ designates the analyte was below the lower limit of quantification but above the limit of

532 detection, while "nd" indicates the analyte was below the limit of detection. NA indicates the

533 analyte was not measured in this sample. 
US Environmental Protection Agency, 2011. Questions and Answers on Dispersants. http://www.epa.gov/bpspill/dispersants-qanda.html. (May 8, 2012)

Anderson, B.S., Arenella-Parkerson, D., Phillips, B.M., Tjeerdema, R.S., Crane, D., 2009. Preliminary investigation of the effects of dispersed Prudhoe Bay Crude Oil on developing topsmelt embryos, Atherinops affinis. Environ. Pollut. 157, 1058-1061.

Backe, W.J., Ort, C., Brewer, A.J., Field, J.A., 2011. Analysis of Androgenic Steroids in Environmental Waters by Large-Volume Injection Liquid Chromatography Tandem Mass Spectrometry. Analytical Chemistry 83, 2622-2630.

Baczko, K., Chasseray, X., Larpent, C., 2001. Synthesis and surfactant properties of symmetric and unsymmetric sulfosuccinic diesters, Aerosol-OT homologues. Journal of the Chemical Society, Perkin Transactions 2, 2179-2188.

Borisov, O.V., Ji, J.A., Wang, Y.J., Vega, F., Ling, V.T., 2011. Toward Understanding Molecular Heterogeneity of Polysorbates by Application of Liquid Chromatography-Mass Spectrometry with Computer-Aided Data Analysis. Analytical Chemistry 83, 3934-3942. Busetti, F., Backe, W., Bendixen, N., Maier, U., Place, B., Giger, W., Field, J., 2012. Trace analysis of environmental matrices by large-volume injection and liquid chromatography-mass spectrometry. Analytical and Bioanalytical Chemistry 402, 175-186.

Campo, P., Venosa, A.D., Suidan, M.T., 2013. Biodegradability of Corexit 9500 and Dispersed South Louisiana Crude Oil at 5 and $25^{\circ} \mathrm{C}$. Environmental Science \& Technology 47, 1960-1967. Health Canada, 1999. Therapeutic Products Programme Guideline: Validation of Analytical Procedures: Methodology. Ottawa, Ontario. Chiaia, A.C., Banta-Green, C., Field, J., 2008. Eliminating Solid Phase Extraction with LargeVolume Injection LC/MS/MS: Analysis of Illicit and Legal Drugs and Human Urine Indicators in US Wastewaters. Environmental Science \& Technology 42, 8841-8848.

Crescenzi, C., Di Corcia, A., Samperi, R., Marcomini, A., 1995. Determination of Nonionic Polyethoxylate Surfactants in Environmental Waters by Liquid Chromatography/Electrospray Mass Spectrometry. Analytical Chemistry 67, 1797-1804.

National Commission on the BP Deepwater Horizon Oil Spill and Offshore Drilling, 2011. The Use of Surface and Subsea Dispersants During the BP Deepwater Horizon Oil Spill.

George-Ares, A., Clark, J.R., 2000. Aquatic toxicity of two Corexit ${ }^{\circledR}$ dispersants. Chemosphere 40, 897-906.

Goodrich, M.S., Melancon, M.J., Davis, R.A., Lech, J.J., 1991. The Toxicity, Bioaccumulation, Metabolism and Elimination of Dioctyl Sodium Sulfosuccinate (DSS) in Rainbow Trout (Oncorhynchus Mykiss). Water Research 25, 119-124.

United States Geological Survey, 2010. Determination of the Anionic Surfactant Di(Ethylhexyl) Sodium Sulfosuccinate in Water Samples Collected from Gulf of Mexico Coastal Waters Before and After Landfall of Oil from the Deepwater Horizon Oil Spill, May to October, 2010. Grimalt, S., Pozo, Ó.J., Marín, J.M., Sancho, J.V., Hernández, F., 2005. Evaluation of Different Quantitative Approaches for the Determination of Noneasily Ionizable Molecules by Different Atmospheric Pressure Interfaces Used in Liquid Chromatography Tandem Mass Spectrometry: Abamectin as Case of Study. Journal of the American Society for Mass Spectrometry 16, 16191630 . 
Hales, S.G., 1993. Biodegradation of the anionic surfactant dialkyl sulphosuccinate.

580 Environmental Toxicology and Chemistry 12, 1821-1828.

581 Hayworth, J.S., Clement, T.P., 2012. Provenance of Corexit-related chemical constituents found in nearshore and inland Gulf Coast waters. Marine Pollution Bulletin 64, 2005-2014. Joye, S.B., MacDonald, I.R., Leifer, I., Asper, V., 2011. Magnitude and oxidation potential of hydrocarbon gases released from the BP oil well blowout. Nature Geosci 4, 160-164.

Kerwin, B.A., 2008. Polysorbates 20 and 80 used in the formulation of protein biotherapeutics: Structure and degradation pathways. Journal of Pharmaceutical Sciences 97, 2924-2935. Kujawinski, E.B., Soule, M.C.K., Valentine, D.L., Boysen, A.K., Longnecker, K., Redmond, M.C., 2011. Fate of Dispersants Associated with the Deepwater Horizon Oil Spill. Environmental Science \& Technology 45, 1298-1306. Mathew, J., Schroeder, D.L., Zintek, L.B., Schupp, C.R., Kosempa, M.G., Zachary, A.M., Schupp, G.C., Wesolowski, D.J., 2012. Dioctyl sulfosuccinate analysis in near-shore Gulf of Mexico water by direct-injection liquid chromatography-tandem mass spectrometry. Journal of Chromatography A 1231, 46-51.

Operational Science Advisory Team, Unified Area Command, 2010. Summary Report for SubSea and Sub-Surface Oil and Dispersant Detection: Sampling and Monitoring.

Petrovic, M., Barceló, D., 2001. Analysis of ethoxylated nonionic surfactants and their metabolites by liquid chromatography/atmospheric pressure ionization mass spectrometry. Journal of Mass Spectrometry 36, 1173-1185.

Petrovic, M., Fernández-Alba, A.R., Borrull, F., Marce, R.M., Mazo, E.G., Barceló, D., 2002. Occurrence and distribution of nonionic surfactants, their degradation products, and linear alkylbenzene sulfonates in coastal waters and sediments in Spain. Environmental Toxicology and Chemistry 21, 37-46.

Place, B., Anderson, B., Mekebri, A., Furlong, E.T., Gray, J.L., Tjeerdema, R., Field, J., 2010. A Role for Analytical Chemistry in Advancing our Understanding of the Occurrence, Fate, and Effects of Corexit Oil Dispersants. Environmental Science \& Technology 44, 6016-6018. Powley, C.R., George, S.W., Ryan, T.W., Buck, R.C., 2005. Matrix Effect-Free Analytical Methods for Determination of Perfluorinated Carboxylic Acids in Environmental Matrixes. Analytical Chemistry 77, 6353-6358.

Pozo, O.J., Deventer, K., Van Eenoo, P., Delbeke, F.T., 2008. Efficient Approach for the Comprehensive Detection of Unknown Anabolic Steroids and Metabolites in Human Urine by Liquid Chromatography-Electrospray-Tandem Mass Spectrometry. Analytical Chemistry 80, 1709-1720.

Ramirez, C., Batchu, S., Gardinali, P., 2013. High sensitivity liquid chromatography tandem mass spectrometric methods for the analysis of dioctyl sulfosuccinate in different stages of an oil spill response monitoring effort. Analytical and Bioanalytical Chemistry 405, 4167-4175. Tellingen, O.v., Beijnen, J.H., Verweij, J., Scherrenburg, E.J., Nooijen, W.J., Sparreboom, A., 1999. Rapid Esterase-sensitive Breakdown of Polysorbate 80 and Its Impact on the Plasma Pharmacokinetics of Docetaxel and Metabolites in Mice. Clinical Cancer Research 5, 2918-2924. Wooten, K., Finch, B., Smith, P., 2012. Embryotoxicity of Corexit 9500 in mallard ducks (Anas platyrhynchos). Ecotoxicology 21, 662-666. 